\title{
Comparison of PET/CT with Sequential PET/MRI Using an MR-Compatible Mobile PET System
}

\author{
Ryusuke Nakamoto, Yuji Nakamoto, Takayoshi Ishimori, Yasutaka Fushimi, Aki Kido, and Kaori Togashi \\ Department of Diagnostic Imaging and Nuclear Medicine, Kyoto University Graduate School of Medicine, Kyoto, Japan
}

\begin{abstract}
The current study tested a newly developed flexible PET (fxPET) scanner prototype. This fXPET system involves dual arc-shaped detectors based on silicon photomultipliers that are designed to fit existing MRI devices, allowing us to obtain fused PET and MR images by sequential PET and MR scanning. This prospective study sought to evaluate the image quality, lesion detection rate, and quantitative values of fxPET in comparison with conventional wholebody (WB) PET and to assess the accuracy of registration. Methods: Seventeen patients with suspected or known malignant tumors were analyzed. Approximately $1 \mathrm{~h}$ after intravenous injection of ${ }^{18} \mathrm{~F}-\mathrm{FDG}$, WB PET/CT was performed, followed by fXPET and MRI. For reconstruction of fxPET images, MRI-based attenuation correction was applied. The quality of fXPET images was visually assessed, and the number of detected lesions was compared between the 2 imaging methods. SUV $\max$ and maximum average SUV within a $1 \mathrm{~cm}^{3}$ spheric volume $\left(\mathrm{SUV}_{\text {peak }}\right)$ of lesions were also compared. In addition, the magnitude of misregistration between fXPET and MR images was evaluated. Results: The image quality of fxPET was acceptable for diagnosis of malignant tumors. There was no significant difference in detectability of malignant lesions between fXPET and WB PET $(P>0.05)$. However, the fXPET system did not exhibit superior performance to the WB PET system. There were strong positive correlations between the 2 imaging modalities in $\operatorname{SUV}_{\text {max }}(\rho=0.88)$ and $\operatorname{SUV}_{\text {peak }}(\rho=0.81) . \mathrm{SUV}_{\text {max }}$ and SUV $\mathrm{Seak}_{\text {peak }}$ measured with fXPET were approximately 1.1-fold greater than measured with WB PET. The average misregistration between fXPET and MR images was $5.5 \pm 3.4 \mathrm{~mm}$. Conclusion: Our preliminary data indicate that running an fxPET scanner near an existing MRI system provides visually and quantitatively acceptable fused PET/MR images for diagnosis of malignant lesions.
\end{abstract}

Key Words: flexible PET scanner; mobile PET; PET/MRI; MRI

J Nucl Med 2018; 59:846-851

DOI: 10.2967/jnumed.117.197665

\section{H}

ybrid PET/MRI is a recent advance in multimodality imaging, providing both anatomic and functional information $(1,2)$, and has several important advantages over PET/CT systems. First, MRI is considered superior to CT for showing soft-tissue contrast and is regarded as the first-line imaging procedure in oncology for

Received Jun. 21, 2017; revision accepted Oct. 12, 2017.

For correspondence or reprints contact: Yuji Nakamoto, Department of Diagnostic Imaging and Nuclear Medicine, Kyoto University Graduate School of Medicine, 54 Shogoin-kawahara-cho, Sakyo-Ku, Kyoto 6068507, Japan.

E-mail: ynakamo1@kuhp.kyoto-u.ac.jp

Published online Nov. 2, 2017.

COPYRIGHT (C 2018 by the Society of Nuclear Medicine and Molecular Imaging. soft-tissue regions (e.g., tumors in the brain, the head-and-neck region, the musculoskeletal region, and the pelvis) (3). Second, MRI can yield functional information about perfusion, diffusion, and metabolism (2). Third, the use of MRI instead of CT for attenuation correction reduces radiation dose, particularly in patients undergoing repeated PET studies (4,5). In addition, integrated PET/MRI systems that perform simultaneous PET and MRI acquisitions afford significantly more accurate registration than the sequential scanning of conventional PET/CT scanners (6). Precise registration between PET and anatomic MRI is important for diagnosing pathologic conditions, avoiding errors in interpretation, planning surgery, and delineating radiation therapy margins (6). PET/MRI scanners have recently been developed by several vendors, and an increasing number of studies using these systems have been published. However, PET/MRI scanners are not widely available, because the costs (including the cost of facilities) are far greater than those of PET/CT. To overcome these limitations, a multimodality-compatible prototype called the flexible PET system (fxPET) has been developed. This new device is a prototype of an MRI-compatible PET scanner with silicon photomultiplier array-based depth-ofinteraction and time-of-flight (TOF)-capable detectors (7). This device is designed to fit existing MRI devices, allowing fused PET and MR images to be obtained by sequential PET and MR scanning.

The purpose of this prospective study was to evaluate the image quality, lesion detection rate, and quantitative values of fxPET, compared with conventional whole-body (WB) PET, and to assess the accuracy of registration.

\section{MATERIALS AND METHODS}

\section{Patients}

In total, 17 patients ( 9 male and 8 female) with suspected or known malignant tumors who underwent fxPET scanning between February 2015 and May 2015 were analyzed. All patients underwent a dualimaging, single-injection protocol consisting of PET/CT and subsequent fxPET/MRI on the same day. The institutional review board approved the study, and all subjects gave written informed consent.

\section{Description of fxPET System}

The fxPET scanner (Fig. 1) consists of 2 detector units that enable the fitting of beds or other imaging modalities and patients of various body sizes (8). The dual arc-shaped detector heads can be arranged in various configurations, including top-bottom and left-right, depending on the purpose of imaging. Each detector unit consists of 18 detector modules in the transaxial direction and 3 rings in the axial direction, with a detector ring diameter of $778 \mathrm{~mm}$ and an axial extent of $150 \mathrm{~mm}$. The detector block comprises 4-layer depth-of-interaction crystal blocks of lutetium oxyorthosilicate crystals (Hitachi Chemical), a light guide, and a 64-channel MRI-compatible silicon photomultiplier array (Hamamatsu Photonics). The coincidence timing resolution of the system is approximately 500 ps. As shown in Figure 1, 


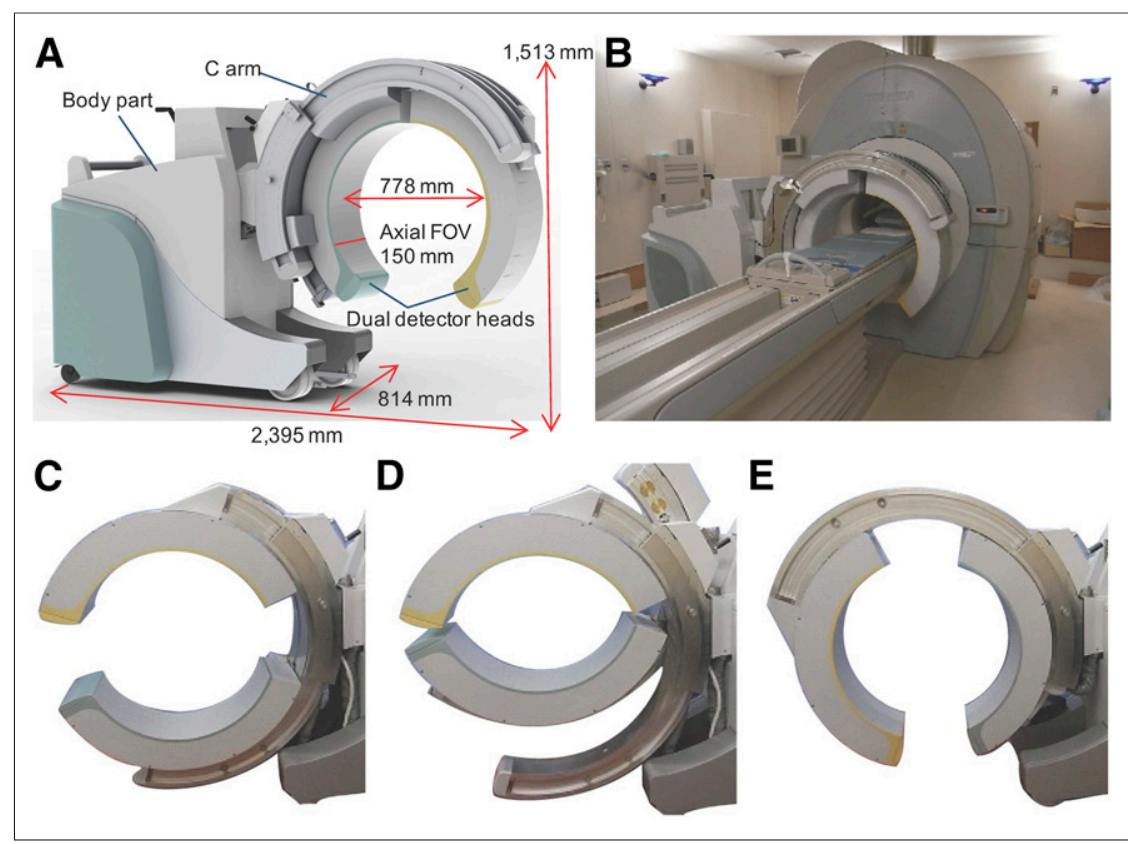

FIGURE 1. (A) Appearance of fxPET scanner. (B) Device is designed to fit existing MRI systems. (C-E) Dual arc-shaped detector heads can be arranged in various configurations: top-bottom (C), near-mode top-bottom (D), and left-right (E), depending on purpose of imaging. FOV = field of view.

the fXPET scanner is a partial-ring scanner rather than a conventional full-ring scanner, and its limited angular coverage results in missed line-of-responses in sinogram space. Degradation of image quality caused by data loss due to incomplete coincidence data was confirmed by a previous phantom study and was found to be reduced by using TOF information and more suitable list-mode reconstruction

TABLE 1

Specifications and Characteristics of fXPET Scanner

\begin{tabular}{|c|c|}
\hline System description & Description value \\
\hline Crystal material & LGSO $\left(\mathrm{Lu}_{1.8} \mathrm{Gd}_{0.2} \mathrm{SiO}_{5}: \mathrm{Ce}\right)$ \\
\hline Crystal size $\left(\mathrm{mm}^{3}\right)$ & $2.9 \times 2.9 \times 20$ \\
\hline Crystal block & $\begin{array}{l}\text { Four layers of } 16 \times 16 \text { arrays } \\
\text { ( } 256 \text { crystal elements) }\end{array}$ \\
\hline $\begin{array}{l}\text { No. of detector } \\
\text { block modules }\end{array}$ & 108 (18 modules $\times 3$ rings $\times 2$ heads $)$ \\
\hline No. of crystals & 27,648 \\
\hline $\begin{array}{l}\text { Detector ring } \\
\quad \text { diameter }(\mathrm{mm})\end{array}$ & 778 \\
\hline Axial FOV (mm) & 150 \\
\hline $\begin{array}{l}\text { Transaxial } \\
\text { FOV (mm) }\end{array}$ & 720 \\
\hline $\begin{array}{l}\text { Energy } \\
\text { resolution (\%) }\end{array}$ & 12.8 \\
\hline $\begin{array}{l}\text { Temporal resolution } \\
\text { of TOF (ps) }\end{array}$ & 500 \\
\hline $\begin{array}{l}\text { Spatial } \\
\quad \text { resolution }(\mathrm{mm})\end{array}$ & $<2.5$ \\
\hline
\end{tabular}

FOV $=$ field of view; LGSO = lutetium oxyorthosilicate. algorithms $(8,9)$. Each crystal is $20 \mathrm{~mm}$ long, with a cross-sectional area of $2.9 \times 2.9 \mathrm{~mm}$ and 4-layer depth-of-interaction capability. The spatial resolution measured with an ${ }^{18} \mathrm{~F}$ point source was estimated to be less than $2.5 \mathrm{~mm}$. The main specifications of the fxPET system are summarized in Table 1 .

Several phantom studies were conducted to prepare for the clinical fxPET/MRI study. A National Electrical Manufacturers Association image-quality phantom study found that the fxPET device could clearly depict the smallest sphere $(10 \mathrm{~mm})(7)$. Another preclinical study using an MR phantom found that installation of the fxPET scanner on a 1.5-T MRI system had no adverse effect on MR images (10). In addition, an experimental study found that the 1.5-T MRI system had no adverse effects on fxPET images (10).

\section{Conventional PET/CT and fXPET/MRI}

An intravenous injection of ${ }^{18} \mathrm{~F}-\mathrm{FDG}$ ( $3.7 \mathrm{MBq} / \mathrm{kg}$ of weight) was followed by WB PET/CT $1 \mathrm{~h}$ later, using a combined PET/CT scanner (Discovery ST Elite or Discovery IQ; GE Healthcare) for 2-3 min/bed position. Patients then underwent fxPET scanning for $10 \mathrm{~min} / \mathrm{bed}$ position, followed by MR scanning for $20 \mathrm{~min}$ (mean, $17 \mathrm{~min}$; range, 10-25 min). To reduce the time needed for fxPET/MR scanning, the scan range of fXPET was limited to one bed position (axial field of view, $150 \mathrm{~mm}$ ). Therefore, the fxPET scan range was determined according to the site of the primary tumors (neck, upper abdomen, pelvis, or musculoskeletal region) using information from CT, MRI, and WB PET/CT findings obtained before fxPET scanning. All fxPET scanning was performed with dual arc-shaped detectors arranged in a top-bottom configuration (Fig. 1C). The central angle of the detector was $135^{\circ}$, and the detector pair covered $270^{\circ}$ of $360^{\circ}$ (Supplemental Fig. 1; supplemental materials are available at http://jnm.snmjournals.org). A larger MR scan range was used to include the whole scan range of fxPET. The mean duration between ${ }^{18} \mathrm{~F}-\mathrm{FDG}$ injection and the start of fxPET scanning was 114 min (range, 92-161 min). Table 2 summarizes the differences in acquisition conditions and system sensitivity between the fxPET and WB PET/CT systems. No patients had a plasma glucose level higher than $200 \mathrm{mg} / \mathrm{dL}$. WB PET images were attenuation-corrected using CT data and reconstructed using a 3-dimensional ordered-subsets expectation-maximization algorithm called VUE Point Plus (Discovery ST Elite: 14 subsets, 2 iterations, a matrix size of $128 \times 128$, a voxel size of $4.7 \times 4.7 \times 3.3 \mathrm{~mm}$, and postfiltering at $5 \mathrm{~mm}$ in full width at half maximum; Discovery IQ: 12 subsets, 4 iterations, a matrix size of $192 \times 192$, a voxel size of $3.3 \times 3.3$ $\times 3.3 \mathrm{~mm}$, and postfiltering at $5 \mathrm{~mm}$ in full width at half maximum). All acquisition fxPET data were reconstructed with a dynamic row-action maximum-likelihood algorithm (11) (128 subsets, 1 iteration, a matrix size of $240 \times 240 \times 50$, a voxel size of $3.0 \times 3.0 \times 3.0 \mathrm{~mm}$, and relaxation control parameter $\beta=30$ with postfiltering at $5 \mathrm{~mm}$ in full width at half maximum). The MR scanner was a 1.5-T system (EXCELART Vantage; Toshiba Medical Systems Corp.). Diagnostic MRI sequences (including T1-weighted imaging, T2-weighted imaging, T2-weighted short- $\tau$ inversion recovery, and diffusion-weighted imaging) were determined according to the site of primary tumors. We applied a newly developed method of MRI-based attenuation correction to the fxPET images $(12,13)$. Conventional segmentation methods of MRI-based attenuation correction ignore the presence of bony structures and have been found to 
TABLE 2

Difference in Acquisition Conditions and System Sensitivity Between fxPET and WB PET/CT Systems

\begin{tabular}{llll}
\hline \multicolumn{1}{c}{ Parameter } & \multicolumn{1}{c}{ fXPET } & \multicolumn{1}{c}{ Discovery STE } & Discovery IQ \\
\hline $\begin{array}{l}\text { Mean time between }{ }^{18} \mathrm{~F}-\mathrm{FDG} \\
\text { injection and start of scan }\end{array}$ & $114 \mathrm{~min}$ (range, 92-161 min) & $58 \mathrm{~min}$ (range, 49-67 min) & $60 \mathrm{~min}$ (range, 54-68 min) \\
Acquisition time & $10 \mathrm{~min} / \mathrm{bed}$ position & $2-3 \mathrm{~min} / \mathrm{bed} \mathrm{position}$ & $2-3 \mathrm{~min} / \mathrm{bed} \mathrm{position}$ \\
Sensitivity (the center) & $2.98 \mathrm{cps} / \mathrm{kBq}$ & $9.0 \mathrm{cps} / \mathrm{kBq}$ & $19.44 \mathrm{cps} / \mathrm{kBq}$ \\
Time-of-flight technology & Available & Not available & Not available \\
Spatial resolution $(\mathrm{mm})$ & $<2.5^{*}$ & $<6.7$ & $<6.2$
\end{tabular}

*Based on iterative point-spread function reconstruction algorithm.

underestimate SUVs in regions containing bone or regions near large bony structures, such as the brain and pelvis (5). In contrast, the new method generates a distribution of attenuation-correction factors by considering bone attenuation using diagnostic T1-weighted images. The parameters of the axial T1-weighted images were determined according to the site scanned: a matrix size of $192-576 \times 288-576$, a field of view of $220-350 \mathrm{~mm} \times 220-500 \mathrm{~mm}$, a slice thickness of 4.0 or $5.0 \mathrm{~mm}$ with a 0 - or 1-mm gap, a repetition time of $272-570 \mathrm{~ms}$, an echo time of $4.8-15 \mathrm{~ms}$, and a flip angle of $70^{\circ}$ or $90^{\circ}$. The attenuation map for the MRI table was obtained using the background activity of lutetium oxyorthosilicate scintillators. The method of scatter corrections was TOF single-scatter simulation (14).

\section{Visual Analysis}

Evaluation of Image Quality. The quality of fxPET images was visually compared with that of WB PET images by 2 board-certified nuclear medicine physicians who did not know the clinical information or the findings of other clinical images. They used a 4-point grading scale: 1, poor for diagnosis; 2, acceptable for diagnosis but inferior to WB PET; 3, comparable to WB PET; and 4, superior to WB PET. The degree of interobserver agreement was evaluated using weighted к-statistics. $\kappa$-values of $0.81-1.00,0.61-0.8,0.41-0.60,0.21-0.40$, and $0-0.2$ were defined as indicating almost perfect, substantial, moderate, fair, and slight agreement, respectively (15).

Evaluation of Lesion Detection Rate. The number of lesions detected on fxPET images was compared with the number detected on WB PET images based on the final diagnosis reached through consensus by 2 board-certified nuclear medicine physicians. Lesions less than $5 \mathrm{~mm}$ in diameter on CT or MRI were excluded from the analysis. Focal moderate to intense ${ }^{18}$ F-FDG uptake, compared with the surrounding tissue, was regarded as positive. If lesions were so close to each other that uptake in the individual lesions could not be distinguished, they were counted as one lesion. The gold standard for the final diagnosis was histopathologic findings (biopsy or surgery), serial radiologic follow-up (CT or MRI or WB PET/CT) revealing further metastatic lesions, or disappearance of metastatic lesions after systemic therapy or clinical follow-up for at least 6 mo (16). Both fXPET and WB PET images were evaluated using OsiriX MD (Pixmeo).

\section{Quantitative Analysis}

$\mathrm{SUV}_{\text {max }}$ and maximum average $\mathrm{SUV}$ within a $1 \mathrm{~cm}^{3}$ spheric volume $\left(\mathrm{SUV}_{\text {peak }}\right)$ of lesions were measured for both fxPET and WB PET images (17). The $\mathrm{SUV}_{\text {max }}$ and $\mathrm{SUV}_{\text {peak }}$ were available only for lesions that met the gold standard, were entirely within the field of view, and could be visually detected on both fxPET and WB PET images $(n=$ 28). Pulmonary lesions and lesions containing wide lung fields in the same axial cross section were excluded because the MRI-based attenuation-correction method did not account for attenuation of lung fields.

\section{Registration Accuracy}

To obtain accurate registration, fxPET and MR images were fused using software in combination with 3-dimensional positional information obtained from an optical Polaris camera (Northern Digital Inc.). This optical tracking system provides accurate, real-time measurement data on 3-dimensional position and orientation tracking of markers attached to the surface of both modalities for accurate image fusion (Fig. 2). Before the clinical study, a study using a water phantom and point sources was performed to evaluate the accuracy of image registration. The results revealed that the average misregistration between fxPET and MR images was approximately $2 \mathrm{~mm}$.

In the clinical study, the registration accuracy of clinical fxPET/ MR images was retrospectively evaluated by one observer using OsiriX MD. The spatial coordinates $(x, y$, and $z$ ) of the visually estimated centers of the lesions were determined for fxPET and MR images independently by calculating the middle slice for each section for all 3 planes (18). The coordinate differential in the 3 axes was determined, and $\Delta x$ was defined as coordinate $x$-axis fxPET coordinate $x$-axis MRI. The same process was used for the $y$ - and $z$-axes. The total difference between the lesion centers on fxPET and MRI was determined by the following formula: difference $=\left(\Delta x^{2}+\right.$ $\left.\Delta y^{2}+\Delta z^{2}\right)^{0.5}(19)$. The same 28 lesions included in the quantitative analysis were analyzed. To avoid inaccurate measurement, 6 lesions from a patient who could not remain still during the fxPET/MRI

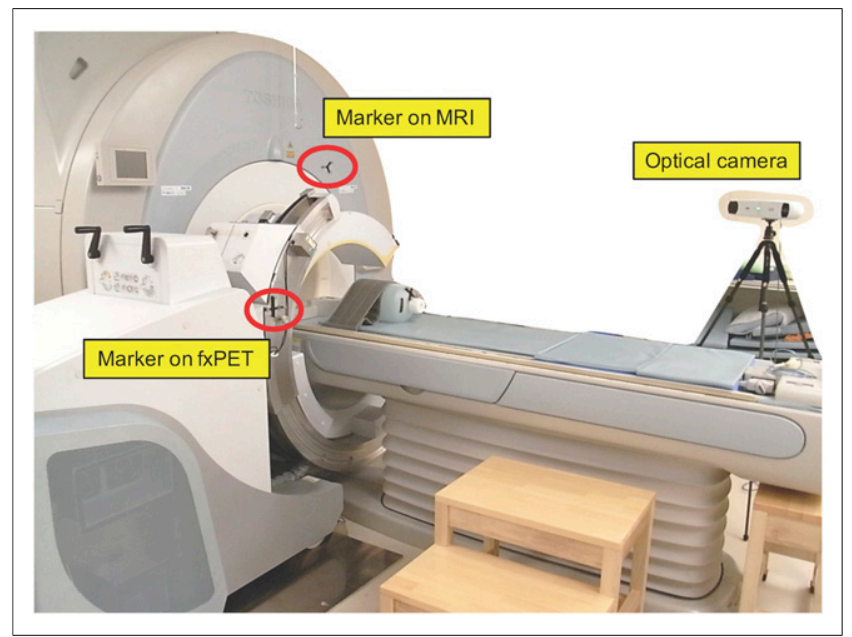

FIGURE 2. Method of fxPET/MR image registration. Spatial registration of $\mathrm{fXPET} / \mathrm{MRI}$ was performed with optical camera measuring positional information of markers (red circles) labeled on surfaces of both imaging modalities. 
examination were excluded from the analysis. One lesion in which ${ }^{18} \mathrm{~F}-\mathrm{FDG}$ uptake was not homogeneous enough to visually determine the boundaries of the uptake was also excluded. Thus, in total, 21 lesions were finally evaluated for the extent of misregistration on fxPET/MR images.

\section{Statistical Analysis}

The sensitivities of fxPET and WB PET images for lesion detection were compared with the final diagnosis using the McNemar test. The Spearman rank correlation coefficient $(\rho)$ was calculated to correlate the $\mathrm{SUV}_{\text {max }}$ and $\mathrm{SUV}_{\text {peak }}$ of malignant lesions between the 2 scanners. A $P$ value of less than 0.05 was considered to indicate statistical significance. Prism (version 6; GraphPad Software) for Microsoft Windows and Microsoft Excel 2016 was used for statistical analysis.

\section{RESULTS}

Patient characteristics, including the results of the visual analysis, are shown in Table 3. Figure 3 shows a representative fxPET/MRI study.

On visual assessment of fxPET image quality, the median grade was 2 for both observers (acceptable for diagnosis but inferior to WB PET). The weighted $\kappa$-score for the quantitative scales between the 2 observers for the quality of fxPET images was "substantial" (0.653; 95\% confidence interval, 0.021-1.000).

Forty-one malignant lesions (primary tumors, metastatic lymph nodes, and distant metastases) were detected based on the gold standard. Of these, 35 malignant lesions $(85 \%)$ were detected by fxPET and $36(88 \%)$ by WB PET. The detectability of malignant lesions did not significantly differ between fxPET and WB PET $(P>0.05)$. In addition, there were no false-positive findings in either PET study.

There were strong positive correlations in $\operatorname{SUV}_{\max }(\rho=0.88)$ and $\operatorname{SUV}_{\text {peak }}(\rho=0.81)$ between fxPET and WB PET (Fig. 4). $\mathrm{SUV}_{\max }$ and $\mathrm{SUV}_{\text {peak }}$ were 1.1-fold greater for fxPET than for WB PET/CT.

The average misregistration of the center of lesions measured with fxPET and MRI was $5.5 \pm 3.4 \mathrm{~mm}$ (Table 4). Misregistration was not consistent but was more pronounced in the $z$-axis $(3.3 \pm 2.5 \mathrm{~mm})$ than in the $x$-axis $(1.3 \pm 1.1 \mathrm{~mm})$ or $y$-axis $(2.5 \pm 1.7 \mathrm{~mm})$.

\section{DISCUSSION}

The visual analysis indicated that the fxPET system produces images of acceptable quality for diagnosis of malignant lesions. In addition, the detectability of malignant lesions did not significantly differ between fxPET and WB PET $(P>0.05)$. To our knowledge, this was the first clinical study to evaluate the feasibility of an MRI-compatible mobile PET system, in which PET images can be less expensively fused to MR images, which have higher soft-tissue contrast and more functional information. This system is considered helpful for reducing radiation dose, compared with PET/CT, especially when repeated scans are necessary.

The image quality of fxPET was not better than that of WB PET, although we used a longer acquisition time for fxPET (10 vs. 2-3 $\mathrm{min} / \mathrm{bed}$ ). The most likely cause of the lack of superior performance was degradation of image quality caused by incomplete

TABLE 3

Patient Characteristics and Results of Visual Analysis

\begin{tabular}{|c|c|c|c|c|c|c|c|c|c|c|c|}
\hline \multirow[b]{2}{*}{$\begin{array}{l}\text { Patient } \\
\text { no. }\end{array}$} & \multirow[b]{2}{*}{ Site } & \multirow[b]{2}{*}{ Sex } & \multirow[b]{2}{*}{ Age (y) } & \multirow[b]{2}{*}{ Disease } & \multicolumn{2}{|c|}{$\begin{array}{l}\text { Minutes from injection } \\
\text { to scanning }\end{array}$} & \multicolumn{2}{|c|}{$\begin{array}{l}\text { Image quality on } \\
\text { 4-point scale }\end{array}$} & \multicolumn{3}{|c|}{$\begin{array}{l}\text { No. of detected } \\
\text { lesions }\end{array}$} \\
\hline & & & & & WB PET & fXPET & $\begin{array}{c}\text { Reader } \\
1\end{array}$ & $\begin{array}{l}\text { Reader } \\
\quad 2\end{array}$ & $\begin{array}{c}\text { Gold } \\
\text { standard }\end{array}$ & $\begin{array}{l}\text { WB } \\
\text { PET }\end{array}$ & fXPET \\
\hline 1 & Neck & $M$ & 46 & Bone tumor & 52 & 96 & 2 & 2 & 3 & 3 & 3 \\
\hline 2 & & M & 59 & Malignant lymphoma & 58 & 110 & 2 & 2 & 8 & 7 & 6 \\
\hline 3 & & $M$ & 66 & Tongue cancer & 50 & 92 & 2 & 2 & 1 & 1 & 1 \\
\hline 4 & & M & 76 & Melanoma & 54 & 121 & 2 & 2 & 2 & 2 & 2 \\
\hline 5 & Upper abdomen & $\mathrm{F}$ & 65 & Ovarian cancer & 64 & 126 & 2 & 2 & 0 & 0 & 0 \\
\hline 6 & & M & 70 & Pancreatic cancer & 50 & 106 & 2 & 2 & 1 & 1 & 1 \\
\hline 7 & & M & 74 & Hepatocellular carcinoma & 62 & 99 & 2 & 2 & 1 & 1 & 1 \\
\hline 8 & & M & 77 & Cholangiocarcinoma & 62 & 104 & 2 & 2 & 5 & 4 & 4 \\
\hline 9 & & $\mathrm{~F}$ & 62 & $\begin{array}{l}\text { Pancreatic neuroendocrine } \\
\text { tumor }\end{array}$ & 58 & 101 & 2 & 2 & 1 & 1 & 1 \\
\hline 10 & & $\mathrm{~F}$ & 77 & $\begin{array}{l}\text { Pancreatic neuroendocrine } \\
\text { tumor }\end{array}$ & 49 & 111 & 2 & 2 & 4 & 1 & 1 \\
\hline 11 & Pelvis & $\mathrm{F}$ & 66 & Endometrioid cancer & 62 & 108 & 3 & 3 & 4 & 4 & 4 \\
\hline 12 & & $\mathrm{~F}$ & 30 & Uterine cervical cancer & 67 & 115 & 2 & 1 & 1 & 1 & 1 \\
\hline 13 & & $\mathrm{~F}$ & 68 & Uterine cervical cancer & 66 & 132 & 2 & 2 & 6 & 6 & 6 \\
\hline 14 & & $\mathrm{~F}$ & 52 & Uterine cervical cancer & 60 & 103 & 2 & 2 & 1 & 1 & 1 \\
\hline 15 & & M & 62 & Rectal cancer & 68 & 129 & 2 & 2 & 1 & 1 & 1 \\
\hline 16 & Musculoskeletal & M & 73 & Soft-tissue sarcoma & 64 & 126 & 2 & 2 & 1 & 1 & 1 \\
\hline 17 & & $\mathrm{~F}$ & 39 & Soft-tissue sarcoma & 60 & 161 & 2 & 2 & 1 & 1 & 1 \\
\hline Total & & $9 \mathrm{M}, 8 \mathrm{~F}$ & $62.5 \pm 13.6^{\star}$ & & $59.2 \pm 6.2^{\star}$ & $114.1 \pm 17^{\star}$ & $2^{\dagger}$ & $2^{\dagger}$ & 41 & 36 & 35 \\
\hline
\end{tabular}




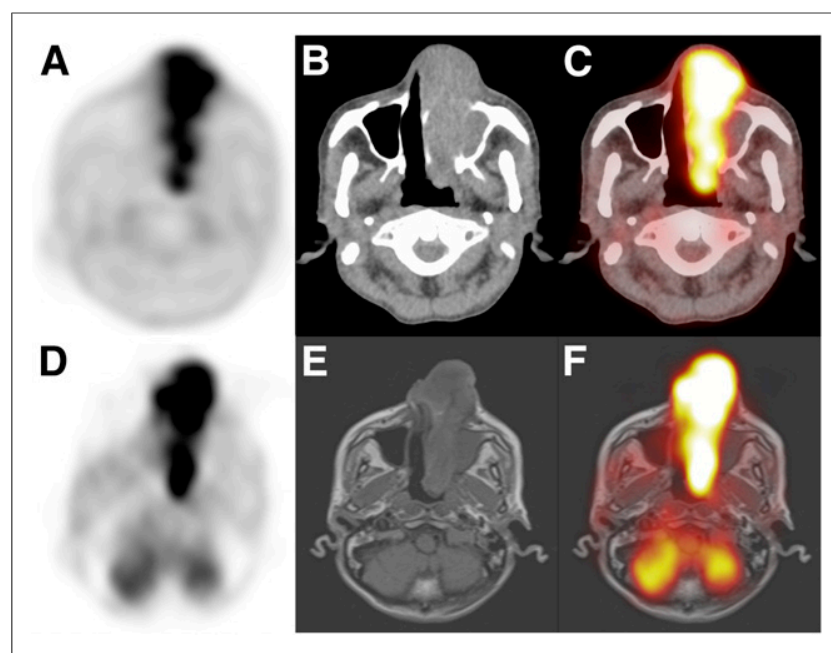

FIGURE 3. Representative case: 76-y-old man with histologically proven nasal mucosal melanoma in left nasal cavity. (A-C) ${ }^{18} \mathrm{~F}-\mathrm{FDG}$ PET/CT scan, including PET (A), CT (B), and fused PET/CT images (C), shows left nasal tumor with focally intense ${ }^{18} \mathrm{~F}-\mathrm{FDG}$ uptake. (D-F) fXPET/MR scan, including PET (D), T1-weighted MR (E), and fused fXPET/MR (F), also depicts tumor clearly.

coincidence data derived from the partial-ring detector. Two studies reported that accurate TOF information improved reconstruction image quality in a partial-ring PET scanner with no detector rotation $(8,9)$. Therefore, the technology of TOF was added to the fxPET system to improve image quality, although the image quality of fXPET was not superior to that of commercial WB PET scanners that do not have TOF capability. Another potential explanation is that the reconstruction conditions of fxPET images may not have been sufficiently optimized. To further improve image quality, data preprocessing and reconstruction methods dedicated to partial-ring TOF PET are now under development.

The $\mathrm{SUV}_{\text {max }}$ and $\mathrm{SUV}_{\text {peak }}$ of lesions were 1.1-fold greater for fxPET than for WB PET/CT. Several factors may have contributed to this difference. First, the different duration after administration of the PET tracers (59 vs. $114 \mathrm{~min}$ ) may have influenced the difference in SUVs, because the radioactive uptake of a lesion has been reported to continue increasing after injection in many cases $(20,21)$. Thus, a longer uptake phase could result in overestimation, rather than underestimation, of the quantitative values of malignant lesions on fxPET images. Second, fxPET images were reconstructed without accounting for attenuation from MR coils. This fact may have contributed to decreased SUVs for lesions on fxPET images. Third, attenuation correction for the MRI table may have been inaccurate, because the materials of the table were unknown. Finally, data loss due to obtaining incomplete coincidence data from the dual arc-shaped detectors arranged in a top-bottom configuration may have degraded quantitative accuracy, although fxPET images were reconstructed using TOF information to reduce the effects of incomplete projection data. The difference between CT-based and MRI-based attenuation correction was unlikely to have affected the difference in SUVs between the 2 scanners in this study, because a newly developed method of attenuation correction that accounts for attenuation of bony structures was applied to the fxPET images.

The average misregistration between fxPET and MR images was $5.5 \mathrm{~mm}$, and the performance of the system in the clinical study was inferior to that in the phantom study (by $\sim 2 \mathrm{~mm}$ ). The cause of the discrepancy between the 2 results may be related to the effects of physiologic organ motion, such as respiratory motion and intestinal peristalsis, caused by the different acquisition timing between fxPET and MRI (18). Rakheja et al. reported that the average misregistration between PET and T1-weighted MR images on a hybrid PET/MRI system was $2.4 \mathrm{~mm}$ (6). Their PET/ MRI system exhibited more accurate spatial registration than the system in the current study. A possible explanation for this difference is that their PET/MRI system could perform simultaneous acquisition whereas the current system required PET and MRI data to be collected sequentially. Nevertheless, our data suggest that acceptably accurate fused fxPET/MR images were obtained from the current system, comparable with the lesion misregistration reported in PET/CT images in previous studies $(4.1 \pm 4.2 \mathrm{~mm})(6)$.

This pilot study revealed several problems with our prototype mobile PET system that need to be solved to improve its performance. Nevertheless, we believe that the fxPET scanner would be an attractive imaging tool, especially from the standpoint of cost. In any facilities having a state-of-art MR system, fused PET and MR images can be obtained at low cost, although the scanning is not simultaneous but sequential. Also, because of the wide inner diameter of the fxPET system, it can use the standard radiofrequency coils of MR scanning if a CT attenuation map of them is prepared and incorporated into reconstruction of the fxPET images. For this reason, it is not necessary to prepare dedicated radiofrequency coils for combined PET/MRI. In addition, this system could be installed for other devices, such as CT or radiation therapy equipment.

The current study had several limitations that should be considered. First, the number of enrolled patients was relatively small $(n=17)$. A prospective study with more patients is warranted to clarify the clinical impact of the fxPET scanner. Second, although 3-T MRI is now becoming the clinical standard, this pilot study was performed with a 1.5-T MRI system. It is physically fXPET. 
TABLE 4

Difference Between Registrations of Lesion Center Measured in 3 Axes with fxPET and MRI

\begin{tabular}{lc}
\hline Axis & Difference \\
\hline$x$ & $1.3 \pm 1.1$ \\
$y$ & $2.5 \pm 1.7$ \\
$z$ & $3.3 \pm 2.5$ \\
Total & $5.5 \pm 3.4$ \\
& \\
\hline Data are mean millimeters \pm SD $(n=21)$. \\
\hline
\end{tabular}

feasible to install the fxPET system near a 3-T system, and further studies are needed to confirm whether fxPET combined with a 3-T system can provide high-quality fused PET/MR images without any PET and MRI artifacts. Third, because of the relatively long acquisition time of fXPET (10 $\mathrm{min} / \mathrm{bed}$ position), the scan range of fxPET/MRI was limited to only one bed position (axial field of view, $150 \mathrm{~mm}$ ). To widen the scan range of fxPET/MRI, we plan to evaluate the extent to which we can shorten the fxPET acquisition time without degrading the quality of the fxPET images using listmode fxPET data. Because the spatial resolution of the fxPET scanner is high $(<2.5 \mathrm{~mm}$ in full width at half maximum), we expect that the detectability of small lesions (e.g., lymph node metastases) would be better than with a conventional PET scanner.

\section{CONCLUSION}

Our preliminary data indicate that an fXPET scanner placed near an existing MRI system provided fused PET/MR images that are visually and quantitatively acceptable for the diagnosis of malignant lesions.

\section{DISCLOSURE}

Financial support was provided by Shimadzu Corp., Kyoto, Japan. No other potential conflict of interest relevant to this article was reported.

\section{ACKNOWLEDGMENTS}

We thank Kyoko Takakura, RT, for her excellent clinical assistance, and we thank Yoshiyuki Yamakawa, Masafumi Furuta, Masanobu Satoh, Tetsuya Kobayashi, Junichi Ohi, and Keishi Kitamura for their technical advice. This study was based on results obtained from a project commissioned by the New Energy and Industrial Technology Development Organization (NEDO).

\section{REFERENCES}

1. Minamimoto R, Levin C, Jamali M, et al. Improvements in PET image quality in time of flight (TOF) simultaneous PET/MRI. Mol Imaging Biol. 2016;18:776-781.

2. Pichler BJ, Kolb A, Nagele T, Schlemmer HP. PET/MRI: paving the way for the next generation of clinical multimodality imaging applications. J Nucl Med. 2010; 51:333-336.

3. Drzezga A, Souvatzoglou M, Eiber M, et al. First clinical experience with integrated whole-body PET/MR: comparison to PET/CT in patients with oncologic diagnoses. J Nucl Med. 2012;53:845-855.

4. Chawla SC, Federman N, Zhang D, et al. Estimated cumulative radiation dose from PET/CT in children with malignancies: a 5-year retrospective review. Pediatr Radiol. 2010;40:681-686.

5. Varoquaux A, Rager O, Poncet A, et al. Detection and quantification of focal uptake in head and neck tumours: ${ }^{18} \mathrm{~F}-\mathrm{FDG}$ PET/MR versus PET/CT. Eur J Nucl Med Mol Imaging. 2014;41:462-475.

6. Rakheja R, DeMello L, Chandarana H, et al. Comparison of the accuracy of PET/CT and PET/MRI spatial registration of multiple metastatic lesions. AJR. 2013;201:1120-1123.

7. Yamakawa Y, Kobayashi T, Furuta M, et al. Development of a dual-head mobile DOITOF PET system having multi-modality compatibility. In: Nuclear Science Symposium and Medical Imaging Conference (NSS/MIC). Piscataway, NJ: IEEE; 2014:1-3

8. Kobayashi T, Kitamura K. Design considerations for a partial-ring, multi-modal compatible whole-body TOF PET scanner: flexible PET. In: Nuclear Science Symposium and Medical Imaging Conference (NSS/MIC). Piscataway, NJ: IEEE; 2012:2807-2812.

9. Surti S, Karp JS. Design considerations for a limited angle, dedicated breast, TOF PET scanner. Phys Med Biol. 2008;53:2911-2921.

10. Furuta M, Satoh M, Ohi J, et al. Development of a proof of concept system for multi-modal compatible PET: flexible PET. In: Nuclear Science Symposium and Medical Imaging Conference (NSS/MIC). Piscataway, NJ: IEEE; 2013:1-4

11. Nakayama T, Kudo H. Derivation and implementation of ordered-subsets algorithms for list-mode PET data. In: Nuclear Science Symposium Conference Record. Piscataway, NJ: IEEE; 2005;4:5.

12. Kawaguchi H, Obata $\mathrm{T}$, Sano H, et al. A hybrid-segmentation atlas method to construct the attenuation correction factor for human pelvic PET/MRI. Proc Intl Soc Mag Reson Med. 2016;24:2186.

13. Tanigawa A, Yamaya T, Kawaguchi H, et al. Hybrid segmentation-atlas method for PET-MRI attenuation correction. In: Nuclear Science Symposium and Medical Imaging Conference (NSS/MIC). Piscataway, NJ: IEEE; 2012:2727-2729.

14. Watson C. Extension of single scatter simulation to scatter correction of time-offlight PET. IEEE Trans Nucl Sci. 2007;54:1679-1686.

15. Kundel HL, Polansky M. Measurement of observer agreement 1. Radiology. 2003;228:303-308.

16. Ali TFT. Usefulness of PET-CT in the assessment of suspected recurrent colorectal carcinoma. Egyptian J Radiol Nucl Med. 2012;43:129-137.

17. Boellaard R, Delgado-Bolton R, Oyen WJ, et al. FDG PET/CT: EANM procedure guidelines for tumour imaging: version 2.0. Eur J Nucl Med Mol Imaging. 2015;42:328-354.

18. Nakamoto Y, Tatsumi M, Cohade C, Osman M, Marshall LT, Wahl RL. Accuracy of image fusion of normal upper abdominal organs visualized with PET/CT. Eur J Nucl Med Mol Imaging. 2003;30:597-602.

19. Cohade C, Osman M, Marshall LN, Wahl RN. PET-CT: accuracy of PET and CT spatial registration of lung lesions. Eur J Nucl Med Mol Imaging. 2003;30:721-726.

20. Basu S, Alavi A. Partial volume correction of standardized uptake values and the dual time point in FDG-PET imaging: should these be routinely employed in assessing patients with cancer? Eur J Nucl Med Mol Imaging. 2007;34:1527-1529.

21. Nakamoto Y, Ishimori T, Sano K, et al. Clinical efficacy of dual-phase scanning using ${ }^{68} \mathrm{Ga}$-DOTATOC-PET/CT in the detection of neuroendocrine tumours. Clin Radiol. 2016;71:1069.e1-1069.e5. 